Portland State University

PDXScholar

Electrical and Computer Engineering Faculty

Publications and Presentations

Electrical and Computer Engineering

$1-1-1994$

\title{
Threshold characteristics of multimode semiconductor lasers
}

Lee W. Casperson

Portland State University

Mehdi Khoshnevissan

Follow this and additional works at: https://pdxscholar.library.pdx.edu/ece_fac

Part of the Electrical and Computer Engineering Commons

Let us know how access to this document benefits you.

\section{Citation Details}

Casperson, L. W., \& Khoshnevissan, M. (1994). Threshold characteristics of multimode semiconductor lasers. Journal Of Applied Physics, 75(2), 737.

This Article is brought to you for free and open access. It has been accepted for inclusion in Electrical and Computer Engineering Faculty Publications and Presentations by an authorized administrator of PDXScholar. Please contact us if we can make this document more accessible: pdxscholar@pdx.edu. 


\title{
Threshold characteristics of multimode semiconductor lasers
}

\author{
Lee W. Casperson and Mehdi Khoshnevissan \\ Department of Electrical Engineering, Portland State University, P.O. Box 751, \\ Portland, Oregon 97207-0751
}

\section{(Received 30 July 1993; accepted for publication 8 October 1993)}

\begin{abstract}
The effects of spontaneous emission on the threshold characteristics of multimode laser oscillators with mixed line broadening are considered in detail. Most previous studies of multimode laser operation have emphasized the two limits of homogeneous and inhomogeneous broadening. With homogeneous broadening there is a tendency for oscillation in a single longitudinal mode, while in the inhomogeneous limit oscillation over much of the gain spectrum is expected. All practical lasers are somewhere between these limits, and this study explores the mode characteristics of lasers with mixed line broadening. Special attention is given to the important practical case of semiconductor diode lasers, and band-to-band absorption is fully included.
\end{abstract}

\section{INTRODUCTION}

Most of the basic features of laser oscillation are now well understood. Far below threshold the laser output typically is in the form of a spontaneous emission spectrum that has been filtered to some extent by the cavity-mode resonances of the Fabry-Perot laser resonator. This belowthreshold behavior is nearly independent of whether homogeneous or inhomogeneous line broadening is dominant, and in both cases the spectral width narrows uniformly as the laser is brought closer to the oscillation threshold. Well above threshold the behavior is signifcantly different for the two broadening mechanisms. In the simplest models of homogeneously broadened lasers, it is well known that single-mode operation is expected for operation above threshold. The lowest loss mode clamps the gain and holds all other modes below their oscillation threshold, and this behavior has long been recognized. ${ }^{1}$ Experimentally, it was found that in the vicinity of threshold the output might consist of a large number of cavity modes, and this mode spectrum only reduces to a single mode for operation somewhat above the lasing threshold. ${ }^{2}$ Analytic expressions have been derived for the oscillation linewidths, mode intensities, and overall spectral envelope of the output from homogeneously broadened lasers for all values of the threshold and noise input parameters; and the results are in good agreement with data obtained using semiconductor lasers. ${ }^{3}$

In inhomogeneously broadened lasers the abovethreshold behavior is very different from the behavior for homogencously broadened lasers. After passing through a minimum near the oscillation threshold the mode spectrum rebroadens with increased pumping until it reaches the full inhomogeneous linewidth. This occurs because the saturation by the central modes does not reduce the gain seen by the modes in the wings of the gain profile, and those side modes are eventually able to reach their own lasing threshold. Expressions have also been derived for the linewidths, mode intensities, and spectral profile of inhomogeneously broadened lasers.

While the threshold characteristics of lasers in the limiting cases of homogeneous and inhomogeneous broadening are reasonably well understood, the more practical cases of mixed line broadening have been studied very little. An obvious reason for this more limited effort is the substantial mathematical difficulties that arise for cases of mixed broadening. The possible multimode consequences of mixed line broadening are known for many laser types including semiconductor diode lasers, ${ }^{4-6}$ and very complex semiclassical models for these systems have been developed. The threshold for multimode oscillation has been obtained neglecting spontaneous emission and saturation by the side modes. ${ }^{7}$ However, the particular problem of the effects of spontaneous emission on multimode oscillation in saturating mixed-broadened lasers has been studied very little. One purpose of the present study is to set up and numerically solve a relatively simple model for such systems.

The spontaneous emission noise effects that lead to multimode oscillation are much stronger in some lasers than in others. The threshold characteristics of semiconductor diode lasers, in particular, are strongly influenced by spontaneous emission. The main reason for this sensitivity to noise is the small cross sectional area of the lasing modes and the resulting large fraction of the total spontaneous emission that these modes capture. The large spontaneous emission input is able to drive modes that otherwise would have been below the lasing threshold. The level of spontaneous emission into the spatial modes of a semiconductor laser has been discusssed by several authors. ${ }^{8-26}$ In the limit of homogeneous broadening, the allocation of the spontaneous emission to the various longitudinal modes of a semiconductor laser has also been considered. ${ }^{27,28}$ In this study a unified representation of the spectral content of the spontaneous emission input has been developed for use in the rate equation models. This form is valid for any level of inhomogeneous line broadening and for arbitrary mode frequency spacings.

In practice it is well known that semiconductor lasers often have many weaker satellite modes in addition to the main oscillating mode. Sometimes these satellite modes are sustained at a cw level, and sometimes they are found to be time sharing the total laser output power. In general, one finds that diodes which have stronger satellite modes under $\mathrm{cw}$ conditions also tend to behave more poorly under mod- 
ulation. Hence, it would be highly desirable to have a truly single-mode laser without any satellite modes, and it is important to inquire into the factors that control the strength and number of these side modes.

Because of the importance of semiconductor lasers, the model that is described here has been formulated to explicitly include the possibility of band-to-band absorption. For applications to nonsemiconductor lasers this term can be set to zero. For the inhomogeneous broadening function we have used a simple Gaussian profile, as in most previous studies. The Gaussian is exactly appropriate for Doppler broadened gas lasers, and it also provides a plausible approximation to the statistical distributions that may be used to represent other types of inhomogeneous line broadening. The Gaussian profile is not always a good approximation for the more complicated Fermi-Dirac distributions that are applied to semiconductor lasers, but in this application it has the compelling advantages of being both explicit and simple.

A rate equation model for the mode intensities in a mixed broadened laser is developed in Sec. II. This model is valid for arbitrary levels of pumping and spontaneous emission noise input and for arbitrary values of the longitudinal mode spacing and the homogeneous and inhomogeneous linewidths. Solutions to these equations in the homogeneous and inhomogeneous line broadening limits are reviewed in Secs. III and IV, respectively. Solutions for the general mixed broadening case are discussed in Sec. V. It is found that rapid rebroadening only occurs when the homogeneous linewidth is somewhat less than the inhomogeneous linewidth.

\section{THEORY}

There are several ways in which one can approach the problem of setting up a theoretical model for the behavior of a multimode laser oscillator. All treatments necessarily involve several approximations, and published studies have varicd as to this choice of approximations. The details depend on the specific problem under consideration and the level of rigor that is required. An early single-mode treatment established a basic set of rate equations for a fourlevel laser including an arbitrary degree of inhomogeneous broadening but excluding the spontaneous emission that is added to the laser mode. ${ }^{29}$ In the meantime there have been several analyses showing how to write and solve multimode rate equations, especially for the case of homogeneous broadening. There have also been many investigations into how the spontaneous emission effects should be included. The results of these studies are mostly consistent with the following general rate equation analysis.

The rate equations governing the populations and the intensity in four-level multimode lasers will be written here as

$$
\begin{aligned}
\frac{\partial N_{2}(v, z, t)}{\partial t}= & S_{2}(v)-A_{2} N_{2}(v, z, t)-\left[N_{2}(v, z, t)-N_{1}(v, z, t)\right] \\
& \times \sum_{j} B\left(v, v_{j}\right)\left[I_{j}^{-}(z, t)+I_{j}^{+}(z, t)\right],
\end{aligned}
$$

$$
\begin{aligned}
\frac{\partial N_{1}(v, z, t)}{\partial t}= & S_{1}(v)+A_{21} N_{2}(v, z, t)-A_{1} N_{1}(v, z, t) \\
& +\left[N_{2}(v, z, t)-N_{1}(v, z, t)\right] \sum_{j} B\left(v, v_{j}\right) \\
& \times\left[I_{j}^{-}(z, t)+I_{j}^{+}(z, t)\right] \\
\frac{n}{c} \frac{\partial I_{j}^{ \pm}(z, t)}{\partial t} \pm & \frac{\partial I_{j}^{ \pm}(z, t)}{\partial z} \\
= & h v_{j} I_{j}^{ \pm}(z, t) \int_{-\infty}^{\infty} B\left(v, v_{j}\right)\left[N_{2}(v, z, t)-N_{1}(v, z, t)\right] d v \\
& -\alpha I_{j}^{ \pm}(z, t)+h v_{j} A_{21} C \int_{v_{j}-\Delta v / 2}^{v_{j}+\Delta v / 2} \int_{-\infty}^{\infty} \frac{2}{\pi \Delta v_{h}} \\
& \times \frac{N_{2}(v, z, t)}{1+\left[2\left(v-v_{i}\right) / \Delta v_{h}\right]^{2}} d v d v_{i},
\end{aligned}
$$

where $N_{2}$ and $N_{1}$ are the population densities of the upper and lower states of the laser transition, $S_{2}$ and $S_{1}$ are the corresponding pump rates, $A_{2}$ and $A_{1}$ are the corresponding total Einstein $A$ coefficients for spontaneous decay, $A_{21}$ is the part of the spontaneous decay that goes directly from level 2 to level 1, and $B$ is the Einstein $B$ coefficient for the transition:

$$
B\left(v, v_{j}\right)=\frac{2 B_{0} /\left(\pi \Delta v_{h}\right)}{1+\left[2\left(\nu-v_{j}\right) / \Delta v_{h}\right]^{2}},
$$

where $\Delta v_{h}$ is the homogeneous linewidth. The intensities of the right and left traveling waves corresponding to mode number $j$ are represented by $I_{j}^{+}$and $I_{j}^{-}$, respectively, $n$ is the index of refraction, $\alpha$ is the internal absorption loss coefficient, and $C$ indicates the fraction of the total spontaneous emission that is added to the lasing modes.

The least obvious aspect of the model given above is the spontaneous emission term in Eq. (3). In this new representation the spontaneous emission from an atom with center frequency $v$ is understood to have a Lorentzian distribution about $v$ with a full width at half maximum of $\Delta v_{h}$. Thus the elements after the integral signs in the spontaneous emission term are proportional to the rate at which spontaneous emission from atoms having a center frequency between $v$ and $v+d v$ is added to the laser intensity in the frequency band between $v_{i}$ and $v_{i}+d v_{i}$. After the inner integral is performed, one has an expression proportional to the total spectral density of the spontaneous emission due to all of the emitting atoms. It is useful to associate the spontaneously emitted radiation continuum with the set of discrete cavity modes, and the easiest way to do that is to integrate the emission spectrum over the frequency range between $v_{j}-\Delta v / 2$ and $v_{j}+\Delta v / 2$, where $v_{j}$ is the dominant frequency associated with mode number $j$ and $\Delta v$ is the cavity mode frequency spacing. ${ }^{30}$

The normalization of the spontaneous emission term can be verified by considering the limit of a single-mode homogeneously broadened laser. The limit of homogeneous broadening is obtained by letting the pumping rates 
and population densities become delta functions of frequency centered at the frequency $v_{0}$ and then using the substitutions

$$
\begin{aligned}
& S_{2}(z, t)=\int_{-\infty}^{\infty} S_{2}(v, z, t) d v \\
& S_{1}(z, t)=\int_{-\infty}^{\infty} S_{1}(v, z, t) d v \\
& N_{2}(z, t)=\int_{-\infty}^{\infty} N_{2}(v, z, t) d v \\
& N_{1}(z, t)=\int_{-\infty}^{\infty} N_{1}(v, z, t) d v .
\end{aligned}
$$

In this way Eqs. (1) $-(3)$ reduce to

$$
\begin{aligned}
\frac{\partial N_{2}(z, t)}{\partial t}= & S_{2}-A_{2} N_{2}(z, t)-\left[N_{2}(z, t)-N_{1}(z, t)\right] \\
& \times \sum_{j} B\left(v_{0}, v_{j}\right)\left[I_{j}^{-}(z, t)+I_{j}^{+}(z, t)\right],
\end{aligned}
$$

$$
\begin{aligned}
\frac{\partial N_{1}(z, t)}{\partial t}= & S_{1}+A_{21} N_{2}(z, t)-A_{1} N_{1}(z, t) \\
& +\left[N_{2}(z, t)-N_{1}(z, t)\right] \sum_{j} B\left(v_{0}, v_{j}\right) \\
& \times\left[I_{j}^{-}(z, t)+I_{j}^{+}(z, t)\right],
\end{aligned}
$$

$$
\begin{aligned}
& \frac{n}{c} \frac{\partial I_{j}^{ \pm}(z, t)}{\partial t} \pm \frac{\partial I_{j}^{ \pm}(z, t)}{\partial z} \\
& =h v_{j} I_{j}^{ \pm}(z, t) B\left(v_{0}, v_{j}\right)\left[N_{2}(z, t)-N_{1}(z, t)\right]-\alpha I_{j}^{ \pm}(z, t) \\
& \quad+h v_{j} A_{21} C \int_{v_{j}-\Delta v / 2}^{v_{j}+\Delta v / 2} \frac{2}{\pi \Delta v_{h}} \\
& \quad \times \frac{N_{2}(z, t)}{1+\left[2\left(v_{0}-v_{i}\right) / \Delta v_{h}\right]^{2}} d v_{i}
\end{aligned}
$$

In the limit of closely spaced modes, the spontaneous emission term in Eq. (11) is similar in effect to the formulations in Refs. 27 and 28. In the opposite limit of singlemode operation, the mode spacing $\Delta v$ may be considered to be very large compared to the homogeneous linewidth $\Delta v_{h}$. Then the integral in Eq. (11) simplifies, and Eqs. (9) $-(11)$ reduce to

$$
\begin{aligned}
\frac{\partial N_{2}(z, t)}{\partial t}= & S_{2}-A_{2} N_{2}(z, t)-\left[N_{2}(z, t)-N_{1}(z, t)\right] \\
& \times B\left[I^{-}(z, t)+I^{+}(z, t)\right],
\end{aligned}
$$

$$
\begin{aligned}
\frac{\partial N_{1}(z, t)}{\partial t}= & S_{1}+A_{21} N_{2}(z, t)-A_{1} N_{1}(z, t) \\
& +\left[N_{2}(z, t)-N_{1}(z, t)\right] B\left[I^{-}(z, t)+I^{+}(z, t)\right],
\end{aligned}
$$

$$
\begin{aligned}
\frac{n}{c} \frac{\partial I^{ \pm}(z, t)}{\partial t} \pm \frac{\partial I^{ \pm}(z, t)}{\partial z} \\
=h v I^{ \pm}(z, t) B\left[N_{2}(z, t)-N_{1}(z, t)\right]-\alpha I^{ \pm}(z, t) \\
\quad+h v A_{21} C N_{2}(z, t),
\end{aligned}
$$

where the mode index $j$ has now been dropped. This reduced set is in a more familiar form and was given as Eqs. (1) $-(3)$ in Ref. 31.

Diode lasers are for many purposes two level systems, and Eqs. (1)-(3) can be adapted to represent their characteristics. ${ }^{32}$ In the spontancous emission terms, $\mathrm{N}_{2}$ can be replaced by $N_{c}$, the excess above the thermal equilibrium concentration of electrons in the conduction band, and $N_{1}$ can become $N_{v}$, the excess of electrons in the valence band. In the stimulated emission terms, $N_{2}$ and $N_{1}$ are replaced by the total concentrations of electrons in the corresponding bands. Then, with some obvious simplifications for a two-level system, Eqs. (1)-(3) become

$$
\begin{aligned}
\frac{\partial N_{c}(v, z, t)}{\partial t}= & S_{c}(v)-A N_{c}(v, z, t) \\
& -\left[N_{c}(v, z, t)-N_{v}(v, z, t)+\Delta N_{0}(v)\right] \\
& \times \sum_{j} B\left(v, v_{j}\right)\left[I_{j}^{-}(z, t)+I_{j}^{+}(z, t)\right],
\end{aligned}
$$

$$
\begin{aligned}
& \frac{\partial N_{v}(v, z, t)}{\partial t}= S_{v}(v)+A N_{c}(v, z, t) \\
&+\left[N_{c}(v, z, t)-N_{v}(v, z, t)+\Delta N_{0}(v)\right] \\
& \times \sum_{j} B\left(v, v_{j}\right)\left[I_{j}^{-}(z, t)+I_{j}^{+}(z, t)\right], \\
& \frac{n}{c} \frac{\partial I_{j}^{ \pm}(z, t)}{\partial t} \pm \frac{\partial I_{j}^{ \pm}(z, t)}{\partial z} \\
&= h v_{j} I_{j}^{ \pm}(z, t) \int_{-\infty}^{\infty} B\left(v, v_{j}\right)\left[N_{c}(v, z, t)-N_{v}(v, z, t)\right. \\
&\left.+\Delta N_{0}(v)\right] d v-\alpha I_{j}^{ \pm}(z, t)+h v_{j} A C \\
& \quad \times \int_{v_{j}-\Delta v / 2}^{v_{j}+\Delta v / 2} \int_{-\infty}^{\infty} \frac{2}{\pi \Delta v_{h}} \\
& \quad \times \frac{N_{c}(v, z, t)}{1+\left[2\left(v-v_{i}\right) / \Delta v_{h}\right]^{2}} d v d v_{i},
\end{aligned}
$$

where $\Delta N_{0}$ is the thermal equilibrium concentration difference $\left(\Delta N_{0}<0\right)$, and the spontaneous emission coefficient is now simply $A$. If one assumes in addition that charge neutrality is maintained $\left(N_{c}+N_{v}=0\right)$, only two equations are necessary, and these can be written 


$$
\begin{aligned}
\frac{\partial N_{c}(v, z, t)}{\partial t}= & S(v)-A N_{c}(v, z, t)-\left[2 N_{c}(v, z, t)+\Delta N_{0}(v)\right] \\
& \times \sum_{j} B\left(v, v_{j}\right)\left[I_{j}^{-}(z, t)+I_{j}^{+}(z, t)\right]
\end{aligned}
$$

$$
\frac{n}{c} \frac{\partial I_{j}^{ \pm}(z, t)}{\partial t} \pm \frac{\partial I_{j}^{ \pm}(z, t)}{\partial z}
$$

$$
\begin{aligned}
= & h v_{j} I_{j}^{ \pm}(z, t) \int_{-\infty}^{\infty} B\left(v, v_{j}\right)\left[2 N_{c}(v, z, t)+\Delta N_{0}(v)\right] d v \\
& -\alpha I_{j}^{ \pm}(z, t)+h v_{j} A C \int_{v_{j}-\Delta v / 2}^{v_{j}+\Delta v / 2} \int_{-\infty}^{\infty} \frac{2}{\pi \Delta v_{h}} \\
& \times \frac{N_{c}(v, z, t)}{1+\left[2\left(v-v_{i}\right) / \Delta v_{h}\right]^{2}} d v d v_{i} .
\end{aligned}
$$

Our main interest here is in the steady-state lasing behavior, and for steady-state operation Eqs. (18) and (19) become

$$
\begin{aligned}
& 0=S(v)-A N_{c}(v, z)-\left[2 N_{c}(v, z)+\dot{\Delta} N_{0}(v)\right] \\
& \times \sum_{j} B\left(v, v_{j}\right)\left[I_{j}^{-}(z)+I_{j}^{+}(z)\right], \\
& \pm \frac{d I_{j}^{ \pm}(z)}{d z}=h v_{j} I_{j}^{ \pm}(z) \int_{-\infty}^{\infty} B\left(v, v_{j}\right) \\
& \times\left[2 N_{c}(v, z)+\Delta N_{0}(v)\right] d v-\alpha I_{j}^{ \pm}(z) \\
& +h v_{j} A C \int_{v_{j}-\Delta v / 2}^{v_{j}+\Delta v / 2} \int_{-\infty}^{\infty} \frac{2}{\pi \Delta v_{h}} \\
& \times \frac{N_{c}(v, z)}{1+\left[2\left(v-v_{i}\right) / \Delta v_{h}\right]^{2}} d v d v_{i} .
\end{aligned}
$$

Equation (20) can be solved for the excess of electrons in the conduction band, and the result is

$$
N_{c}(v, z)=\frac{S(v)-\Delta N_{0}(v) \Sigma_{j} B\left(v, v_{j}\right)\left[I_{j}^{-}(z)+I_{j}^{+}(z)\right]}{A+2 \Sigma_{j} B\left(v, v_{j}\right)\left[I_{j}^{-}(z)+I_{j}^{+}(z)\right]} .
$$

This result may be substituted into Eq. (21), and one obtains

$$
\begin{aligned}
\pm \frac{d I_{j}^{ \pm}(z)}{d z}= & h v_{j} I_{j}^{ \pm}(z) \int_{-\infty}^{\infty} B\left(v, v_{j}\right) \frac{2 S(v) / A+\Delta N_{0}(v)}{1+(2 / A) \Sigma_{j} B\left(v, v_{j}\right)\left[I_{j}^{-}(z)+I_{j}^{+}(z)\right]} d v-\alpha I_{j}^{ \pm}(z) \\
& +\frac{h v_{j} A C}{2} \int_{v_{j}-\Delta v / 2}^{v_{j}+\Delta v / 2} \int_{-\infty}^{\infty} \frac{2 /\left(\pi \Delta v_{h}\right)}{1+\left[2\left(v-v_{i}\right) / \Delta v_{h}\right]^{2}}\left(\frac{2 S(v) / A+\Delta N_{0}(v)}{1+(2 / A) \Sigma_{j} B\left(v, v_{j}\right)\left[I_{j}^{-}(z)+I_{j}^{+}(z)\right]}-\Delta N_{0}\right) d v d v_{i} .
\end{aligned}
$$

Equation (23) can also be written

$$
\begin{aligned}
\pm \frac{d I_{j}^{ \pm}(z)}{d z}= & \frac{2 h v_{j} B_{0}}{\pi \Delta v_{h}} I_{j}^{ \pm}(z) \int_{-\infty}^{\infty} \frac{1}{1+\left(y-y_{j}\right)^{2}} \frac{2 S(y) / A+\Delta N_{0}(y)}{1+\Sigma_{n}\left\{\left[s I_{n}^{-}(z)+s I_{n}^{+}(z)\right] /\left[1+\left(y-y_{n}\right)^{2}\right]\right\}} d y-\alpha I_{j}^{ \pm}(z) \\
& +\frac{h v_{j} A C}{2 \pi} \int_{y_{j}-\Delta y / 2}^{y_{j}+\Delta y / 2} \int_{-\infty}^{\infty} \frac{1}{1+\left(y-y_{i}\right)^{2}}\left(\frac{2 S(y) / A+\Delta N_{0}(y)}{1+\Sigma_{n}\left\{\left[s I_{n}^{-}(z)+s I_{n}^{+}(z)\right] /\left[1+\left(y-y_{n}\right)^{2}\right]\right\}}-\Delta N_{0}(y)\right) d y d y_{i},
\end{aligned}
$$

where we have used the definition of the Einstein $B$ coefficient given in Eq. (4) together with the normalized frequencies $y=2\left(v-v_{0}\right) / \Delta v_{h}$ and $y_{j}=2\left(v_{j}-v_{0}\right) / \Delta v_{h}$ and the saturation parameter $s=4 B_{0} /\left(A \pi \Delta v_{h}\right)$. The frequency $v_{0}$ is a characteristic frequency for the transition. It is convenient to introduce the normalized intensity $X_{j}=s I_{j}$, and then Eq. (24) reduces to

$$
\begin{aligned}
\pm \frac{d X_{j}^{ \pm}(z)}{d z}= & \frac{2 h v_{j} B_{0}}{\pi \Delta v_{h}} X_{j}^{ \pm}(z) \int_{-\infty}^{\infty} \frac{1}{1+\left(y-y_{j}\right)^{2}} \frac{2 S(y) / A+\Delta N_{0}(y)}{1+\Sigma_{n}\left\{\left[X_{n}^{-}(z)+X_{n}^{+}(z)\right] /\left[1+\left(y-y_{n}\right)^{2}\right]\right\}} d y-\alpha X_{j}^{ \pm}(z) \\
& +\frac{2 h v_{j} B_{0} C}{\pi^{2} \Delta v_{h}} \int_{y_{j}-\Delta y / 2}^{y_{j}+\Delta y / 2} \int_{-\infty}^{\infty} \frac{1}{1+\left(y-y_{i}\right)^{2}}\left(\frac{2 S(y) / A+\Delta N_{0}(y)}{1+\Sigma_{n}\left\{\left[X_{n}^{-}(z)+X_{n}^{+}(z)\right] /\left[1+\left(y-y_{n}\right)^{2}\right]\right\}}-\Delta N_{0}(y)\right) d y d y_{i}
\end{aligned}
$$

At this point it is necessary to specify a form for the inhomogeneous spectral distributions. The actual spectral distributions for semiconductor lasers can be somewhat complicated, and the reader may use whatever distribution is deemed appropriate. As mentioned above, we use here a Gaussian distribution as often encountered in laser studies. Thus, it is assumed that the pump and thermal equilibrium concentration difference distributions are given by

$$
\begin{aligned}
& S(y)=S^{\prime} \epsilon \exp \left(-\epsilon^{2} y^{2}\right) / \pi^{1 / 2}, \\
& \Delta N_{0}(y)=\Delta N_{0}^{\prime} \epsilon \exp \left(-\epsilon^{2} y^{2}\right) / \pi^{1 / 2},
\end{aligned}
$$

where the natural damping ratio is ${ }^{29}$ 


$$
\epsilon=\left(\Delta v_{h} / \Delta v_{d}\right)\left(\log _{e} 2\right)^{1 / 2}
$$

With these substitutions Eq. (25) becomes

$$
\begin{aligned}
\pm \frac{d X_{j}^{ \pm}(z)}{d z}= & \frac{2 h v_{j} \epsilon B_{0}}{\pi^{3 / 2} \Delta v_{h}} X_{j}^{ \pm}(z)\left(\frac{2 S^{\prime}}{A}+\Delta N_{0}^{\prime}\right) \int_{-\infty}^{\infty} \frac{\exp \left(-\epsilon^{2} y^{2}\right)}{1+\left(y-y_{j}\right)^{2}} \frac{1}{1+\Sigma_{n}\left\{\left[X_{n}^{-}(z)+X_{n}^{+}(z)\right] /\left[1+\left(y-y_{n}\right)^{2}\right]\right\}} d y-\alpha X_{j}^{ \pm}(z) \\
& +\frac{2 h v_{j} \epsilon B_{0} C}{\pi^{5 / 2} \Delta v_{h}}\left(2 S^{\prime} / A+\Delta N_{0}^{\prime}\right) \int_{y_{j}-\Delta y / 2}^{y_{j}+\Delta y / 2} \int_{-\infty}^{\infty} \frac{\exp \left(-\epsilon^{2} y^{2}\right)}{1+\left(y-y_{i}\right)^{2}} \frac{1}{1+\Sigma_{n}\left\{\left[X_{n}^{-}(z)+X_{n}^{+}(z)\right] /\left[1+\left(y-y_{n}\right)^{2}\right]\right\}} d y d y_{i} \\
& -\frac{2 h v_{j} \epsilon B_{0} C}{\pi^{3 / 2} \Delta v_{h}} \Delta N_{0}^{\prime} \int_{y_{j}-\Delta y / 2}^{y_{j}+\Delta y / 2} \int_{-\infty}^{\infty} \frac{\exp \left(-\epsilon^{2} y^{2}\right)}{1+\left(y-y_{i}\right)^{2}} d y d y_{i} .
\end{aligned}
$$

It is now convenient to introduce the line center unsaturated gain

$$
g_{0}=\frac{4 h v_{j} \in B_{0} S^{\prime}}{\pi^{3 / 2} \Delta v_{h} A} \int_{-\infty}^{\infty} \frac{\exp \left(-\epsilon^{2} y^{2}\right)}{1+y^{2}} d y
$$

and the unsaturated band-to-band absorption

$$
g^{\prime}=-\frac{2 h v_{j} \in B_{0} \Delta N_{0}^{\prime}}{\pi^{3 / 2} \Delta v_{h}} \int_{-\infty}^{\infty} \frac{\exp \left(-\epsilon^{2} y^{2}\right)}{1+y^{2}} d y .
$$

With these substitutions Eq. (29) becomes

$$
\begin{aligned}
\pm \frac{d X_{j}^{ \pm}(z)}{d z}= & \left(g_{0}-g^{\prime}\right) X_{j}^{ \pm}(z) \int_{-\infty}^{\infty} \frac{\exp \left(-\epsilon^{2} y^{2}\right)}{1+\left(y-y_{j}\right)^{2}} \frac{1}{1+\Sigma_{n}\left\{\left[X_{n}^{-}(z)+X_{n}^{+}(z)\right] /\left[1+\left(y-y_{n}\right)^{2}\right]\right\}} d y / \int_{-\infty}^{\infty} \frac{\exp \left(-\epsilon^{2} y^{2}\right)}{1+y^{2}} d y \\
& +\left(g_{0}-g^{\prime}\right) \frac{C}{\pi} \int_{y_{j}-\Delta y / 2}^{y_{j}+\Delta y / 2} \int_{-\infty}^{\infty} \frac{\exp \left(-\epsilon^{2} y^{2}\right)}{1+\left(y-y_{i}\right)^{2}} \frac{1}{1+\Sigma_{n}\left\{\left[X_{n}^{-}(z)+X_{n}^{+}(z)\right] /\left[1+\left(y-y_{n}\right)^{2}\right]\right\}} d y d y_{i} / \\
& \int_{-\infty}^{\infty} \frac{\exp \left(-\epsilon^{2} y^{2}\right)}{1+y^{2}} d y+g^{\prime} \frac{C}{\pi} \int_{y_{j}-\Delta y / 2}^{y_{j}+\Delta y / 2} \int_{-\infty}^{\infty} \frac{\exp \left(-\epsilon^{2} y^{2}\right)}{1+\left(y-y_{i}\right)^{2}} d y d y_{i} / \int_{-\infty}^{\infty} \frac{\exp \left(-\epsilon^{2} y^{2}\right)}{1+y^{2}} d y-\alpha X_{j}^{ \pm}(z)
\end{aligned}
$$

If the gain per pass is small, both $X_{j}^{+}(z)$ and $X_{j}^{-}(z)$ may be approximated by a single parameter $X_{j}(z)$. It is also now possible to assume that the mirror losses are uniformly distributed over the round trip amplifier path length of $2 l$. With these substitutions the rate of change of intensity with distance around the laser can be written

$$
\begin{aligned}
\frac{d X_{j}(z)}{d z}= & \left(g_{0}-g^{\prime}\right) X_{j}(z) \int_{-\infty}^{\infty} \frac{\exp \left(-\epsilon^{2} y^{2}\right)}{1+\left(y-y_{j}\right)^{2}} \frac{1}{1+\Sigma_{n}\left\{2 X_{n}(z) /\left[1+\left(y-y_{n}\right)^{2}\right]\right\}} d y / \int_{-\infty}^{\infty} \frac{\exp \left(-\epsilon^{2} y^{2}\right)}{1+y^{2}} d y+\left(g_{0}-g^{\prime}\right) \frac{C}{\pi} \\
& \times \int_{y_{j}-\Delta y / 2}^{y_{j}+\Delta y / 2} \int_{-\infty}^{\infty} \frac{\exp \left(-\epsilon^{2} y^{2}\right)}{1+\left(y-y_{i}\right)^{2}} \frac{1}{1+\Sigma_{n}\left\{2 X_{n}(z) /\left[1+\left(y-y_{n}\right)^{2}\right]\right\}} d y d y_{i} / \int_{-\infty}^{\infty} \frac{\exp \left(-\epsilon^{2} y^{2}\right)}{1+y^{2}} d y \\
& +g^{\prime} \frac{C}{\pi} \int_{y_{j}-\Delta y / 2}^{y_{j}+\Delta y / 2} \int_{-\infty}^{\infty} \frac{\exp \left(-\epsilon^{2} y^{2}\right)}{1+\left(y-y_{i}\right)^{2}} d y d y_{i} / \int_{-\infty}^{\infty} \frac{\exp \left(-\epsilon^{2} y^{2}\right)}{1+y^{2}} d y-\left(\frac{g_{0}}{r}-g^{\prime}\right) X_{j}(z)
\end{aligned}
$$

where $r$ is a threshold parameter which equals the ratio of the unsaturated line center gain to the total cavity losses. The threshold parameter in this case can be written explicitly

$$
r=\frac{2 g_{0} l}{\left(1-R_{l}\right)+\left(1-R_{r}\right)+2 \alpha l+2 g^{\prime} l},
$$

where $R_{l}$ and $R_{r}$ are the left and right mirror reflectivities, respectively.

Equation (33) is the principal result of this section, and it is the basis for all of the following calculations. In our numerical solutions it can be used to track the evolution of the individual mode intensities as they start out from arbitrary assumed initial conditions and approach the steady-state relationship $d X_{j}(z) / d z=0$. In terms of the normalized distance $z^{\prime}=z\left(g_{0}-g^{\prime}\right)$, Eq. (33) can also be written in the more compact form 


$$
\begin{aligned}
\frac{d X_{j}(z)}{d z^{\prime}}= & X_{j}(z) \int_{-\infty}^{\infty} \frac{\exp \left(-\epsilon^{2} y^{2}\right)}{1+\left(y-y_{j}\right)^{2}} \frac{1}{1+\Sigma_{n}\left\{2 X_{n}(z) /\left[1+\left(y-y_{n}\right)^{2}\right]\right\}} d y / \int_{-\infty}^{\infty} \frac{\exp \left(-\epsilon^{2} y^{2}\right)}{1+y^{2}} d y \\
& +\frac{C}{\pi} \int_{y_{j}-\Delta y / 2}^{y_{j}+\Delta y / 2} \int_{-\infty}^{\infty} \frac{\exp \left(-\epsilon^{2} y^{2}\right)}{1+\left(y-y_{i}\right)^{2}} \frac{1}{1+\Sigma_{n}\left\{2 X_{n}(z) /\left[1+\left(y-y_{n}\right)^{2}\right]\right\}} d y d y_{i} / \int_{-\infty}^{\infty} \frac{\exp \left(-\epsilon^{2} y^{2}\right)}{1+y^{2}} d y \\
& +D \int_{y_{j}-\Delta y / 2}^{y_{j}+\Delta y / 2} \int_{-\infty}^{\infty} \frac{\exp \left(-\epsilon^{2} y^{2}\right)}{1+\left(y-y_{i}\right)^{2}} d y d y_{i} / \int_{-\infty}^{\infty} \frac{\exp \left(-\epsilon^{2} y^{2}\right)}{1+y^{2}} d y-E X_{j}(z)
\end{aligned}
$$

where the new parameters $D$ and $E$ are given by

$$
\begin{aligned}
& D=\frac{C / \pi}{\left(g_{0} / g^{\prime}\right)-1}, \\
& E=\frac{r^{-1}-g^{\prime} / g_{0}}{1-\left(g^{\prime} / g_{0}\right)} .
\end{aligned}
$$

Our most general numerical solutions have been obtained using the model given in Eq. (35).

\section{HOMOGENEOUS BROADENING}

In some cases it is possible to obtain explicit analytic solutions for the characteristics of multimode laser oscillators. To illustrate this possibility, we will consider first the special case of homogeneous broadening. If it is assumed that steady-state operation has been achieved, Eq. (33) can be solved for the mode intensity $X_{j}$, and the result is

$$
\begin{aligned}
X_{j}= & {\left[\left(g_{0}-g^{\prime}\right) \frac{C}{\pi} \int_{y_{j}-\Delta y / 2}^{y_{j}+\Delta y / 2} \int_{-\infty}^{\infty} \frac{\exp \left(-\epsilon^{2} y^{2}\right)}{1+\left(y-y_{i}\right)^{2}} \frac{1}{1+\Sigma_{n}\left\{2 X_{n} /\left[1+\left(y-y_{n}\right)^{2}\right]\right\}} d y d y_{i}\right.} \\
& \left.+g^{\prime} \frac{C}{\pi} \int_{y_{j}-\Delta y / 2}^{y_{j}+\Delta y / 2} \int_{-\infty}^{\infty} \frac{\exp \left(-\epsilon^{2} y^{2}\right)}{1+\left(y-y_{i}\right)^{2}} d y d y_{t}\right] /\left[\left(\frac{g_{0}}{r}-g^{\prime}\right) \int_{-\infty}^{\infty} \frac{\exp \left(-\epsilon^{2} y^{2}\right)}{1+y^{2}} d y-\left(g_{0}-g^{\prime}\right)\right. \\
& \left.\times \int_{-\infty}^{\infty} \frac{\exp \left(-\epsilon^{2} y^{2}\right)}{1+\left(y-y_{j}\right)^{2}} \frac{1}{1+\Sigma_{n}\left\{2 X_{n} /\left[1+\left(y-y_{n}\right)^{2}\right]\right\}} d y\right]
\end{aligned}
$$

It will be assumed that inhomogeneous broadening is negligible $(\epsilon>1)$. Thus, the Gaussian functions in Eq. (38) act like delta functions, and this equation reduces further to

$$
\begin{aligned}
X_{j}= & {\left[\left(g_{0}-g^{\prime}\right) \frac{C}{\pi} \int_{y_{j}-\Delta y / 2}^{y_{j}+\Delta y / 2} \frac{1}{1+y_{i}^{2}} \frac{1}{1+\Sigma_{n}\left[2 X_{n} /\left(1+y_{n}^{2}\right)\right]} d y_{i}\right.} \\
& \left.+g^{\prime} \frac{C}{\pi} \int_{y_{j}-\Delta y / 2}^{y_{j}+\Delta y / 2} \frac{1}{1+y_{i}^{2}} d y_{i}\right] /\left[\left(\frac{g_{0}}{r}-g^{\prime}\right)\right. \\
& \left.-\left(g_{0}-g^{\prime}\right) \frac{1}{1+y_{j}^{2}} \frac{1}{1+\Sigma_{n}\left[2 X_{n} /\left(1+y_{n}^{2}\right)\right]}\right]
\end{aligned}
$$

Next, we will assume that the mode spacing is much less than the homogeneous line width $(\Delta y<1)$. In this limit Eq. (39) becomes

$$
\begin{aligned}
X_{j}= & {\left[\left(g_{0}-g^{\prime}\right) \frac{C \Delta y}{\pi} \frac{1}{1+y_{j}^{2}}-\frac{1}{1+\Sigma_{n}\left[2 X_{n} /\left(1+y_{n}^{2}\right)\right]}\right.} \\
& \left.+g^{\prime} \frac{C \Delta y}{\pi} \frac{1}{1+y_{j}^{2}}\right] /\left[\left(\frac{g_{0}}{r}-g^{\prime}\right)-\left(g_{0}-g^{\prime}\right) \frac{1}{1+y_{j}^{2}}\right. \\
& \left.\times \frac{1}{1+\Sigma_{n}\left[2 X_{n} /\left(1+y_{n}^{2}\right)\right]}\right] .
\end{aligned}
$$

Clearing the fractions, this result can also be written

$$
\begin{aligned}
X_{j}= & \frac{C \Delta y}{\pi}\left[g_{0}+g^{\prime} \sum_{n}\left[2 X_{n} /\left(1+y_{n}^{2}\right)\right]\right] /\left[\left(\frac{g_{0}}{r}-g^{\prime}\right)\right. \\
& \left.\times\left(1+y_{j}^{2}\right)\left(1+\sum_{n} \frac{2 X_{n}}{1+y_{n}^{2}}\right)-\left(g_{0}-g^{\prime}\right)\right] .
\end{aligned}
$$

Both sides of Eq. (41) may be multiplied by $2\left(1+y_{j}^{2}\right)^{-1}$, and the result may be summed over $j$ to obtain 


$$
\begin{aligned}
x= & \frac{2 C \Delta y}{\pi} \sum_{j} \frac{1}{1+y_{j}^{2}} \\
& \times \frac{g_{0}+g^{\prime} x}{\left[\left(g_{0} / r\right)-g^{\prime}\right]\left(1+y_{j}^{2}\right)(1+x)-\left(g_{0}-g^{\prime}\right)},
\end{aligned}
$$

where the intensity parameter $x$ has been defined by

$$
x=\sum_{n} \frac{2 X_{n}}{1+y_{n}^{2}} .
$$

With the previous assumption that the mode spacing is much less than the homogeneous linewidth, the summation on the right-hand side of Eq. (42) can be replaced by an integral:

$$
\begin{aligned}
x= & \frac{2 C}{\pi} \int_{-\infty}^{\infty} \frac{1}{1+y_{j}^{2}} \\
& \times \frac{g_{0}+g^{\prime} x}{\left[\left(g_{0} / r\right)-g^{\prime}\right]\left(1+y_{j}^{2}\right)(1+x)-\left(g_{0}-g^{\prime}\right)} d y_{j} .
\end{aligned}
$$

This integral can be performed using the formula ${ }^{33}$

$$
\int_{0}^{\infty} \frac{x^{\mu-1}}{\left(\beta+x^{2}\right)\left(\gamma+x^{2}\right)} d x=\frac{\pi}{2} \frac{\gamma^{\mu / 2-1}-\beta^{\mu / 2-1}}{\beta-\gamma} \operatorname{cosec}\left(\frac{\mu \pi}{2}\right),
$$

and the result can be written

$$
\begin{aligned}
x= & \frac{2 C\left(g_{0}+g^{\prime} x\right)}{g_{0}-g^{\prime}} \\
& \times\left[\left(\frac{\left[\left(g_{0} / r\right)-g^{\prime}\right](1+x)}{\left[\left(g_{0} / r\right)-g^{\prime}\right](1+x)-\left(g_{0}-g^{\prime}\right)}\right)^{1 / 2}-1\right] .
\end{aligned}
$$

This is a cubic equation for $x$, and the solutions can be obtained explicitly. The parameter $x$ can then be used in Eq. (41) to determine the basic features of the laser output.

The total one-way intensity $x_{t}$ in the laser can be obtained by summing Eq. (41) over all of the cavity modes according to

$$
\begin{aligned}
x_{t} & =\sum_{j} X_{j} \\
& =\frac{C \Delta y}{\pi} \sum_{j} \frac{g_{0}+g^{\prime} x}{\left[\left(g_{0} / r\right)-g^{\prime}\right]\left(1+y_{j}^{2}\right)(1+x)-\left(g_{0}-g^{\prime}\right)} .
\end{aligned}
$$

The summation on the right-hand side of Eq. (47) can be replaced by an integral:

$x_{t}=\frac{C}{\pi} \int_{-\infty}^{\infty} \frac{g_{0}+g^{\prime} x}{\left[\left(g_{0} / r\right)-g^{\prime}\right]\left(1+y_{j}^{2}\right)(1+x)-\left(g_{0}-g^{\prime}\right)} d y_{j}$.

This integral can be performed using the formula

$$
\int_{0}^{\infty} \frac{a d x}{a^{2}+x^{2}} d x=\frac{\pi}{2}
$$

and the result can be written

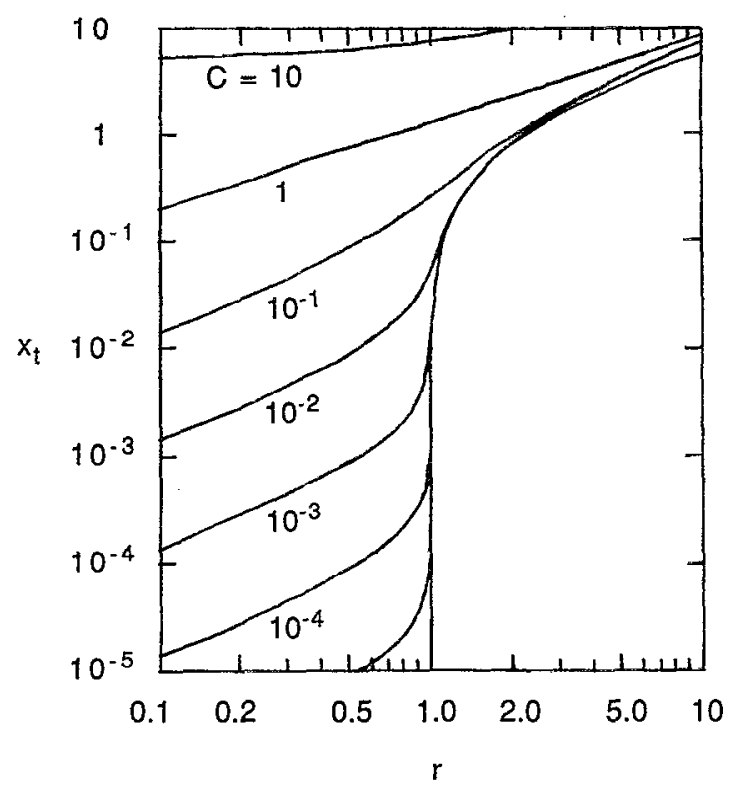

FIG. 1. Total one-way intensity $x_{t}$ in a homogeneously broadened laser as a function of the threshold parameter $r$ for various values of the spontaneous emission parameter $C$.

$$
\begin{aligned}
x_{t}= & C\left(g_{0}+g^{\prime} x\right) /\left\{[ ( \frac { g _ { 0 } } { r } - g ^ { \prime } ) ( 1 + x ) ] ^ { 1 / 2 } \left[\left(\frac{g_{0}}{r}-g^{\prime}\right)\right.\right. \\
& \left.\left.\times(1+x)-\left(g_{0}-g^{\prime}\right)\right]^{1 / 2}\right\} .
\end{aligned}
$$

Equation (50) is an explicit expression for the total one-way intensity when the parameter $x$ is known, and $x$ is obtained from Eq. (46). These results are plotted in Fig. 1 for a wide range of values of $C$ and $r$. The other parameters needed in these calculations include the line center unsaturated gain $g_{0}$ and the unsaturated band-to-band absorption $g^{\prime}$. For the band-to-band absorption we have used the value $g^{\prime}=38 \mathrm{~cm}^{-1}$. The gain $g_{0}$ can be expressed in terms of the threshold parameter $r$ using Eq. (34), and the other parameter values used in this equation include the mirror reflectivities $R_{l}=R_{r}=0.3$, nonsaturating distributed intensity loss rate $\alpha=40 \mathrm{~cm}^{-1}$, and length $l=300 \mu \mathrm{m}$. All of these numerical values are the same as those used and discussed in Ref. 31. It is clear from the figure that for small values of $C$ the intensity increases abruptly when the gain increases past threshold $(r=1)$, while for larger values of $C$ the threshold transition is not so distinct.

It is also of interest to determine the overall width of the multimode laser spectrum. It follows from Eq. (41) that the spectral shape is always Lorentzian, whether the laser is above threshold or not. In real frequency units the full width of the spectrum at half maximum is

$$
\frac{\Delta v_{s}}{\Delta v_{h}}=\left(\frac{\left[\left(g_{0} / r\right)-g^{\prime}\right](1+x)-\left(g_{0}-g^{\prime}\right)}{\left[\left(g_{0} / r\right)-g^{\prime}\right](1+x)}\right)^{1 / 2} .
$$

The values for the parameter $x$ are again obtained as solutions of Eq. (46). In Fig. 2 are plots of the spectral width $\Delta v_{s}$ from Eqs. (51) and (46) for various values of the spontaneous emission noise parameter $C$. The other param- 


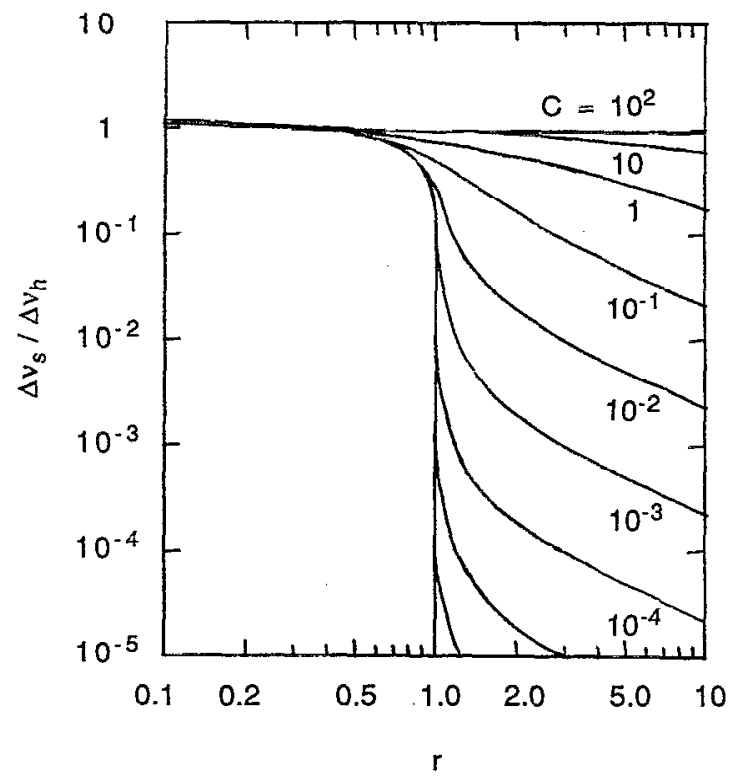

FIG. 2. Normalized width of the mode-spectrum envelope $\Delta v_{s} / \Delta v_{h}$ in a homogeneously broadened laser as a function of the threshold parameter $r$ for various values of $C$.

eter values in these solutions are obtained as described in connection with Fig. 1. It is clear that, as in experimental studies, there is a rapid narrowing of the mode spectrum as the laser begins to operate above threshold.

In Fig. 3 are some typical calculated longitudinal mode spectra, where the normalized mode amplitudes from Eq. (41) are plotted versus the normalized frequency $y=2\left(v-v_{0}\right) / \Delta v_{h}$ for various values of the threshold parameter. In this example the spontaneous emission parameter is $C=10^{-3}$, the mode spacing is $\Delta v_{h} / 20$, and the normalization is such that the center mode amplitude is constant. This figure also shows clearly the rapid line narrowing that occurs near threshold, and the transition is still more abrupt for smaller values of $C$. This means that when $r$ is slightly greater than unity, oscillation is largely confined to a single longitudinal mode.

As a check on these results, we may compare them to the results obtained in a simpler analytic treatment of multimode lasers. Thus, we consider the case of negligible band-to-band absorption $g^{\prime}=0$. In this limit Eq. (46) reduces to

$$
x=2 C\left[\left(\frac{1+x}{1+x-r}\right)^{1 / 2}-1\right],
$$

where the threshold parameter from Eq. (34) is now

$$
r=\frac{2 g_{0} l}{\left(1-R_{l}\right)+\left(1-R_{r}\right)+2 \alpha l} \text {. }
$$

Equation (46) is the same as Eq. (18) of Ref. 3 if $C$ is replaced by $x_{0} / 2$. In the same limit the total intensity $x_{t}$ from Eq. (50) is given more simply as

$$
x_{i}=\sum_{j} X_{j}=\frac{r(1+x)^{-1} C}{\left[1-r(1+x)^{-1}\right]^{1 / 2}}
$$

and this result is equivalent to Eq. (23) of Ref. 3.

The total intensity from Eqs. (52) and (54) can be plotted yielding results that are similar to those in Fig. 1. There are, however, important differences between the predicted intensities with and without band-to-band absorption. For example, for small values of the noise parameter $C$, the above threshold intensity from Eqs. (46) and (50) reduces to $x_{t} \approx 0.80(r-1)$, where the numerical coefficient follows from the assumed numerical values of the various laser parameters employed above. On the other hand, from Eqs. (52) and (54) the total intensity in this limit is $x_{t} \approx 0.50(r-1)$. Thus, the inclusion of band-to-band absorption causes a significant change in the laser's intensity characteristics.

The effects of band-to-band absorption on the mode intensity spectrum of the laser can also be explored. If band-to-band absorption is considered to be negligible, the spectral width from Eq. (51) reduces to

$$
\frac{\Delta v_{s}}{\Delta v_{h}}=\left(\frac{1+x-r}{1+x}\right)^{1 / 2} \text {. }
$$

The longitudinal mode spectral width from Eqs. (52) and (55) is similar to the spectral width given in Fig. 2, but here too the quantitative differences between the models with and without band-to-band absorption are substantial.

\section{INHOMOGENEOUS BROADENING}

Analytic solutions are also possible in the limit of inhomogeneous broadening $(\epsilon<1)$. We will first limit our attention to the interesting case that the mode spacing is small compared to the homogeneous linewidth. Thus the summations in Eq. (38) can be replaced by integrals, and this equation then takes the form

$$
\begin{aligned}
X\left(y_{j}\right) \Delta y= & {\left[\left(g_{0}-g^{\prime}\right) \frac{C}{\pi} \int_{y_{j}-\Delta y / 2}^{y_{j}+\Delta y / 2} \int_{-\infty}^{\infty} \frac{\exp \left(-\epsilon^{2} y^{2}\right)}{1+\left(y-y_{i}\right)^{2}}\left[1 /\left(1+\int_{-\infty}^{\infty} \frac{2 X\left(y_{n}\right) d y_{n}}{1+\left(y-y_{n}\right)^{2}}\right)\right] d y d y_{i}\right.} \\
& \left.+g^{\prime} \frac{C}{\pi} \int_{y_{j}-\Delta y / 2}^{y_{j}+\Delta y / 2} \int_{-\infty}^{\infty} \frac{\exp \left(-\epsilon^{2} y^{2}\right)}{1+\left(y-y_{i}\right)^{2}} d y d y_{i}\right] /\left[\left(\frac{g_{0}}{r}-g^{\prime}\right) \int_{-\infty}^{\infty} \frac{\exp \left(-\epsilon^{2} y^{2}\right)}{1+y^{2}} d y-\left(g_{0}-g^{\prime}\right)\right. \\
& \left.\times \int_{-\infty}^{\infty} \frac{\exp \left(-\epsilon^{2} y^{2}\right)}{1+\left(y-y_{j}\right)^{2}}\left[1 /\left(1+\int_{-\infty}^{\infty} \frac{2 X\left(y_{n}\right) d y_{n}}{1+\left(y-y_{n}\right)^{2}}\right)\right] d y\right]
\end{aligned}
$$


where $X\left(y_{n}\right)$ is the effective spectral density in the vicinity of mode $n$. If the spectral density is uniform over the homogeneous linewidth, then the spectral density can be evaluated at the normalized frequency $y$ and removed from the saturation integrals. These integrals then yield the value $\pi$, and Eq. (56) reduces to

$$
\begin{aligned}
X\left(y_{j}\right) \Delta y= & {\left[\left(g_{0}-g^{\prime}\right) \frac{C}{\pi} \int_{y_{j}-\Delta y / 2}^{y_{j}+\Delta y / 2} \int_{-\infty}^{\infty} \frac{\exp \left(-\epsilon^{2} y^{2}\right)}{1+\left(y-y_{i}\right)^{2}} \frac{1}{1+2 \pi X(y)} d y d y_{i}+g^{\prime} \frac{C}{\pi} \int_{y_{j}-\Delta y / 2}^{y_{j}+\Delta y / 2} \int_{-\infty}^{\infty} \frac{\exp \left(-\epsilon^{2} y^{2}\right)}{1+\left(y-y_{i}\right)^{2}} d y d y_{i}\right] / } \\
& {\left[\left(\frac{g_{0}}{r}-g^{\prime}\right) \int_{-\infty}^{\infty} \frac{\exp \left(-\epsilon^{2} y^{2}\right)}{1+y^{2}} d y-\left(g_{0}-g^{\prime}\right) \int_{-\infty}^{\infty} \frac{\exp \left(-\epsilon^{2} y^{2}\right)}{1+\left(y-y_{j}\right)^{2}} \frac{1}{1+2 \pi X(y)} d y\right] }
\end{aligned}
$$

In the limit of inhomogeneous broadening $(\epsilon<1)$, both the Gaussian functions and the intensity distributions may be removed from the $y$ integrations and the remaining integrals evaluate to $\pi$. Thus Eq. (57) reduces to

$$
X\left(y_{j}\right) \Delta y=\left[\left(g_{0}-g^{\prime}\right) \frac{C}{\pi} \int_{y_{j}-\Delta y / 2}^{y_{j}+\Delta y / 2} \frac{\exp \left(-\epsilon^{2} y_{i}^{2}\right)}{1+2 \pi X\left(y_{i}\right)} d y_{i}+g^{\prime} \frac{C}{\pi} \int_{y_{j}-\Delta y / 2}^{y_{j}+\Delta y / 2} \exp \left(-\epsilon^{2} y_{i}^{2}\right) d y_{i}\right] /\left[\left(\frac{g_{0}}{r}-g^{\prime}\right)-\left(g_{0}-g^{\prime}\right) \frac{\exp \left(-\epsilon^{2} y_{j}^{2}\right)}{1+2 \pi X\left(y_{j}\right)}\right]
$$

For the assumed closely spaced modes the remaining integrals simplify also, and Eq. (58) becomes

$$
\begin{aligned}
X\left(y_{j}\right)= & {\left[\left(g_{0}-g^{\prime}\right) \frac{C}{\pi} \frac{\exp \left(-\epsilon^{2} y_{j}^{2}\right)}{1+2 \pi X\left(y_{j}\right)}+g^{\prime} \frac{C}{\pi} \exp \left(-\epsilon^{2} y_{j}^{2}\right)\right] / } \\
& {\left[\left(\frac{g_{0}}{r}-g^{\prime}\right)-\left(g_{0}-g^{\prime}\right) \frac{\exp \left(-\epsilon^{2} y_{j}^{2}\right)}{1+2 \pi X\left(y_{j}\right)}\right] } \\
= & \frac{C}{\pi}\left[g_{0}+g^{\prime} 2 \pi X\left(y_{j}\right)\right] /\left[\left(\frac{g_{0}}{r}-g^{\prime}\right) \exp \left(\epsilon^{2} y_{j}^{2}\right)\right. \\
& \left.\times\left[1+2 \pi X\left(y_{j}\right)\right]-\left(g_{0}-g^{\prime}\right)\right] .
\end{aligned}
$$

This is a quadratic equation for the spectral density $X\left(y_{j}\right)$, and the various intensity and spectral shape solutions can be readily obtained.

For the special case of no band-to-band absorption $\left(g^{\prime}=0\right)$, Eq. (59) reduces to

$$
X\left(y_{j}\right)=\frac{C / \pi}{r^{-1} \exp \left(\epsilon^{2} y_{j}^{2}\right)\left[1+2 \pi X\left(y_{j}\right)\right]-1},
$$

and this result is equivalent to Eq. (58) of Ref. 3.

\section{MIXED BROADENING}

The previous sections have emphasized the mode behavior of multimode lasers in the limits of homogeneous and inhomogeneous line broadening. These limits are important because most lasers can be identified with one or the other of these classes, and the resulting simplificationsof the analysis can aid in the intuitive understanding of

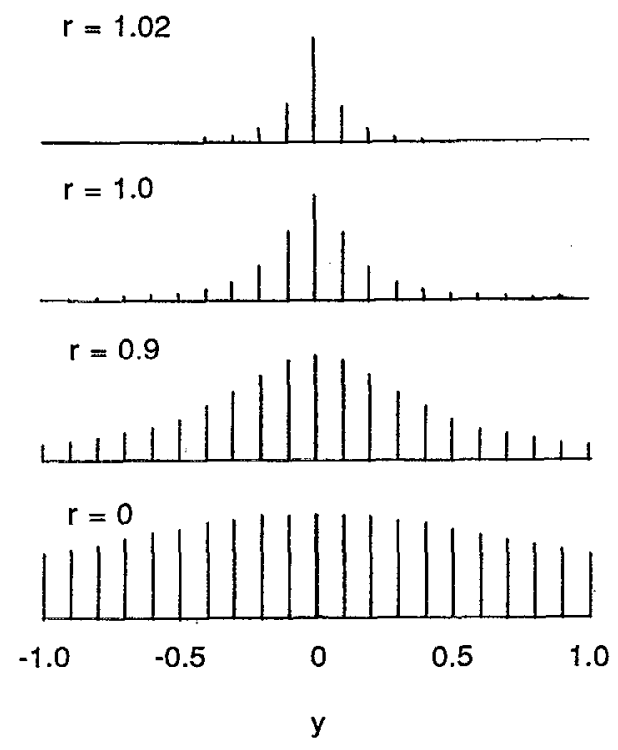

FIG. 3. Normalized longitudinal mode intensities as a function of the normalized frequency $y=2\left(v-v_{0}\right) / \Delta v_{h}$ for various values of the threshold parameter $r$. In this example the spontaneous emission parameter is $C=10^{-3}$ and the mode spacing is $\Delta v_{h} / 20$. 
the laser behavior. On the other hand, some lasers are clearly intermediate between the homogeneous and inhomogeneous limits, and it is important to be able to understand the behavior of these intermediate cases as well. Because of the dramatic differences between the laser behavior in the two limits, it is sometimes difficult to guess the behavior of intermediate systems. Thus there is value in considering representative examples of multimode lasers with mixed line broadening.

Our most general model for the threshold characteris- tics of semiconductor lasers with mixed line broadening has been given above as Eq. (35). The analysis for lasers with mixed broadening is necessarily more complicated than for lasers dominated by homogeneous or inhomogeneous broadening, but there still may be some simplifications that one can make. For example, if the mode spacing is small compared to the homogeneous linewidth, the integrands in the outer spontaneous emission integrals may be evaluated at the mode frequency $v_{j}$, and Eq. (35) reduces to

$$
\begin{aligned}
\frac{d X_{j}(z)}{d z^{\prime}}= & X_{j}(z) \int_{-\infty}^{\infty} \frac{\exp \left(-\epsilon^{2} y^{2}\right)}{1+\left(y-y_{j}\right)^{2}}\left[1 /\left(1+\sum_{n} \frac{2 X_{n}(z)}{1+\left(y-y_{n}\right)^{2}}\right)\right] d y / \int_{-\infty}^{\infty} \frac{\exp \left(-\epsilon^{2} y^{2}\right)}{1+y^{2}} d y \\
& +\frac{C \Delta y}{\pi} \int_{-\infty}^{\infty} \frac{\exp \left(-\epsilon^{2} y^{2}\right)}{1+\left(y-y_{j}\right)^{2}}\left[1 /\left(1+\sum_{n} \frac{2 X_{n}(z)}{1+\left(y-y_{n}\right)^{2}}\right)\right] d y / \int_{-\infty}^{\infty} \frac{\exp \left(-\epsilon^{2} y^{2}\right)}{1+y^{2}} d y \\
& +D \Delta y \int_{-\infty}^{\infty} \frac{\exp \left(-\epsilon^{2} y^{2}\right)}{1+\left(y-y_{j}\right)^{2}} d y / \int_{-\infty}^{\infty} \frac{\exp \left(-\epsilon^{2} y^{2}\right)}{1+y^{2}} d y-E X_{j}(z) .
\end{aligned}
$$

Furthermore, if the mode spectrum were flat compared to the homogeneous linewidth, the spectrum could be replaced by a spectral density function $X\left(y_{j}\right)$ as in Sec. IV above, and the mode summations could be replaced by integrals. Our solutions for mixed broadened lasers have been based on direct numerical solutions of Eq. (61) using a Runge-Kutta method.

In Fig. 4 are plots of the spectral width of a mixed

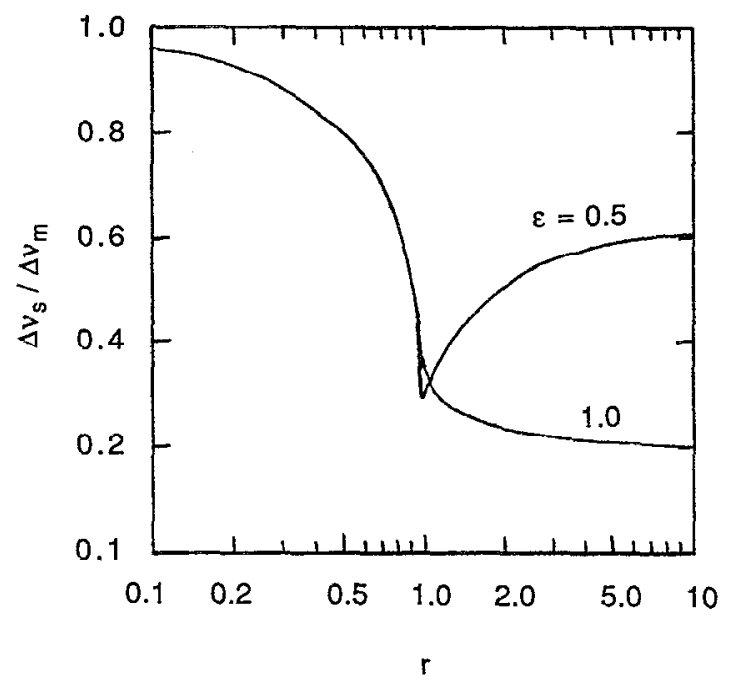

FIG. 4. Normalized width of the mode-spectrum envelope $\Delta v_{s} / \Delta v_{m}$ in a mixed-broadened laser as a function of $r$ for $C=10^{-3}$ and broadening ratios of $\epsilon=0.5$ and $\epsilon=1.0$. broadened semiconductor laser as a function of the threshold parameter $r$ for the natural damping ratios $\epsilon=0.5$ and $\epsilon=1.0$ and with the noise parameter $C=0.001$. The other laser parameters are the same as those employed in Figs. 1-3. The spectral width is normalized to $\Delta v_{m}$, the width of the unsaturated mixed-broadened gain spectrum. It is clear from this figure that the spectral envelope narrows as the laser approaches threshold, but then flattens or rebroadens for operation far above threshold. In these examples, substantial rebroadening occurs for $\epsilon=0.5$ but not for the more homogeneously broadened case $\epsilon=1.0$. This result is consistent with the conclusion reached in a simplified analysis, in which it was shown that spectral rebroadening should only occur for $\epsilon<0.654$. $^{7}$

The corresponding results for the noise parameter $C=0.0001$ are shown in Fig. 5. As with the larger noise parameter employed in Fig. 4, there is again rebroadening for operation far above threshold. However, with the smaller value of $C$ the above threshold linewidth for the homogeneously broadened cases can be smaller than for Fig. 4.

\section{CONCLUSION}

This study has included a detailed discussion of the power characteristics of multimode laser oscillators. Of particular interest has been the threshold behavior of lasers 


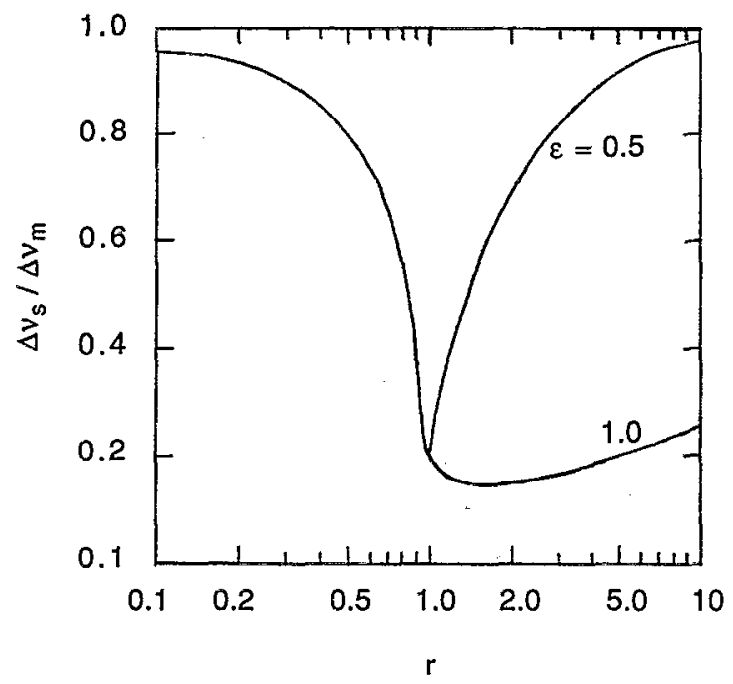

FIG. 5. Normalized width of the mode-spectrum envelope $\Delta v_{s} / \Delta v_{m}$ in a mixed-broadened laser as a function of $r$ for $C=10^{-4}$ and broadening ratios of $\epsilon=0.5$ and $\epsilon=1.0$.

with mixed line broadening. A procedure has been described for representing the spontaneous emission noise in such lasers with arbitrary levels of the saturating intensity of the cavity modes, arbitrary line broadening, and arbitrary mode spacing. It has been shown that the broad below-threshold spectral envelope of the cavity modes narrows dramatically as the threshold parameter $r$ increases to unity. For larger values of $r$ the behavior depends strongly on the ratio of the homogeneous to inhomogeneous linewidths. For large values of this ratio, the linewidth continues to narrow as the laser is operated farther above threshold, while for small values of the ratio there is eventually a rebroadening of the spectral envelope. The threshold properties of semiconductor lasers have been of particular interest, and the model developed here includes an arbitrary level of band-to-band absorption.

\section{ACKNOWLEDGMENTS}

This work was supported in part by the National Science Foundation under Grant No. ECS-9014481 and by Tektronix, Inc.

${ }^{1}$ T. H. Maiman, Phys. Rev. 123, 1145 (1961).

${ }^{2}$ See, for example, W. V. Smith and P. P. Sorokin, The Laser (McGrawHill, New York, 1966), Sec. 3-3.

${ }^{3}$ L. W. Casperson, J. Appl. Phys. 46, 5194 (1975).

${ }^{4}$ B. Zee, IEEE J. Quantum Electron. QE-14, 727 (1978).

${ }^{5}$ M. Yamada and Y. Suematsu, J. Appl. Phys. 52, 2653 (1981).

${ }^{6}$ O. V. Bogdankevich, V. O. Davydov, M. M. Zverev, Y. A. Kudeyarev, and V. N. Faifer, Sov. J. Quantum Electron. 17, 695 (1987).

${ }^{7}$ L. W. Casperson, J. Appl. Phys. 52, 6981 (1981).

${ }^{8}$ T. L. Paoli, IEEE J. Quantum Electron. QE-11, 276 (1975).

${ }^{9}$ Y. Suematsu, S. Akiba, and T. Hong, IEEE J. Quantum Electron. QE-13, 596 (1977).

${ }^{10} \mathrm{~K}$. Petermann, IEEE J. Quantum Electron. QE-15, 566 (1979).

${ }^{11}$ W. Streifer, D. R. Scifres, and R. D. Burnham, Appl. Phys. Lett. 40, 305 (1982).

${ }^{12}$ W. Streifer, D. R. Scifres, and R. D. Burnham, Electron. Lett. 17, 933 (1981).

${ }^{13}$ E. Patzak, Electron. Lett. 18, 278 (1982).

${ }^{14}$ A. Yariv and S. Margalit, IEEE J. Quantum Electron. QE-18, 1831 (1982).

${ }^{15}$ D. Marcuse, Electron. Lett. 18, 920 (1982).

${ }^{16}$ J. Arnaud, Electron. Lett. 19, 688 (1983).

${ }^{17}$ J. Arnaud, Electron. Lett. 19, 798 (1983).

${ }^{18}$ G. P. Agrawal, J. Optical Soc. Am. B 1, 406 (1984).

${ }^{19} \mathrm{M}$. Newstein, IEEE J. Quantum Electron. QE-20, 1270 (1984).

${ }^{20}$ H. A. Haus and S. Kawakami, IEEE J. Quantum Electron. QE-21, 63 (1985).

${ }^{21}$ F. Coste, J. Fesquet, and J. Arnaud, Electron. Lett. 20, 719 (1984).

${ }^{22}$ J. Arnaud, J. Fesquet, F. Coste, and P. Sansonetti, IEEE J. Quantum Electron. QE-21, 603 (1985).

${ }^{23}$ J. Arnaud, IEEE J. Quantum Electron. QE-21, 737 (1985).

${ }^{24} M$. Newstein, IEEE J. Quantum Electron. QE-21, 737 (1985).

${ }^{25}$ J. Arnaud, Electron. Lett. 21, 538 (1985).

${ }^{26}$ M. B. El Mashade and J. Arnaud, IEEE J. Quantum Electron. QE-22, 505 (1986).

${ }^{27}$ T. P. Lee, C. A. Burrus, J. A. Copeland, A. G. Dentai, and D. Marcuse, IEEE J. Quantum Electron. QE-18, 1101 (1982).

${ }^{28}$ D. Marcuse, IEEE J. Quantum Electron. QE-19, 1228 (1983).

${ }^{29}$ E. I. Gordon, A. D. White, and J. D. Rigden, Symposium on Optical Masers (Polytechnic Institute of Brooklyn, 1963), pp. 309-318.

${ }^{30}$ L. W. Casperson, Appl. Optics 14, 299 (1975).

${ }^{31}$ J. Z. Wilcox and L. W. Casperson, J. Appl. Phys. 56, 57 (1984).

${ }^{32} \mathrm{~J}$. Huang and L. W. Casperson, Optical and Quantum Electron. 25, 369 (1993).

${ }^{33}$ I. S. Gradshteyn and I. M. Ryzhik, Table of Integrals, Series, and Products (Academic, New York, 1980), Eq. 3.264-2. 